\title{
Myocardial B cells are a subset of circulating lymphocytes with delayed transit through the heart
}

\author{
Luigi Adamo, ${ }^{1}$ Cibele Rocha-Resende, ${ }^{1}$ Chieh-Yu Lin, ${ }^{2}$ Sarah Evans, ${ }^{1}$ Jesse Williams, ${ }^{3}$ Hao Dun, ${ }^{4}$ \\ Wenjun Li, ${ }^{4}$ Cedric Mpoy, ${ }^{5}$ Prabhakar S. Andhey, ${ }^{2}$ Buck E. Rogers, ${ }^{5}$ Kory Lavine, ${ }^{1}$ Daniel Kreisel,,${ }^{2,4}$ \\ Maxim Artyomov, ${ }^{2}$ Gwendalyn J. Randolph, ${ }^{2}$ and Douglas L. Mann ${ }^{1}$ \\ ${ }^{1}$ Cardiovascular Division, Department of Medicine, and 2Department of Pathology and Immunology, Washington University \\ School of Medicine, St. Louis, Missouri, USA. ${ }^{3}$ Center for Immunology, Department of Integrative Biology and Physiology, \\ University of Minnesota School of Medicine, Minneapolis, Minnesota, USA. ${ }^{4}$ Department of Surgery and ${ }^{5}$ Department of \\ Radiation Oncology, Washington University School of Medicine, St. Louis, Missouri, USA.
}

\begin{abstract}
Current models of B lymphocyte biology posit that B cells continuously recirculate between lymphoid organs, without accumulating in peripheral healthy tissues. Nevertheless, B lymphocytes are one of the most prevalent leukocyte populations in the naive murine heart. To investigate this apparent inconsistency in the literature, we conducted a systematic analysis of myocardial B cell ontogeny, trafficking dynamics, histology, and gene expression patterns. We found that myocardial B cells represent a subpopulation of circulating B cells that make close contact with the microvascular endothelium of the heart and arrest their transit as they pass through the heart. The vast majority ( $>95 \%)$ of myocardial B cells remain intravascular, whereas few $(<5 \%)$ myocardial $B$ cells cross the endothelium into myocardial tissue. Analyses of mice with B cell deficiency or depletion indicated that $B$ cells modulate the myocardial leukocyte pool composition. Analysis of $B$ cell-deficient animals suggested that $B$ cells modulate myocardial growth and contractility. These results transform our current understanding of $B$ cell recirculation in the naive state and reveal a previously unknown relationship between $B$ cells and myocardial physiology. Further work will be needed to assess the relevance of these findings to other organs.
\end{abstract}

Conflict of interest: LA and DLM are cofounders of $\mathrm{i}$-Cordis, LLC, which is focused on the development of B cell-modulating therapies for the treatment of heart failure, and are coinventors on patent W02019028062, "Pirfenidone derivatives for modulation of $B$ lymphocyte activity and organ protection," owned by Washington University in St. Louis.

Copyright: (ㄷ) 2020, American Society for Clinical Investigation.

Submitted: October 31, 2019

Accepted: January 9, 2020

Published: February 13, 2020.

Reference information: /CI Insight. 2020;5(3):e134700.

https://doi.org/10.1172/jci. insight.134700.

\section{Introduction}

B lymphocytes are one of the most prevalent leukocytes in the naive murine heart (1-3). Prior studies have shown that modulating the number and function of myocardial B cells confers cardioprotective effects in both acute and chronic myocardial injury $(2,4-6)$. However, the basic biology of myocardial $\mathrm{B}$ cells in the naive heart is unknown. Indeed, myocardial B cell ontogeny, trafficking dynamics, histology, and identity have not been investigated in the naive heart. Currently, it is not clear why there is a large B cell population in the naive myocardium. According to current models of B cell trafficking, $B$ cells recirculate between primary and secondary lymphoid organs until binding their cognate antigen (7-9). This model does not predict that B cells should accumulate in nonlymphoid healthy tissue. Given that $B$ cells play an important role in myocardial injury $(2,4-6)$, it is crucial to elucidate the biology of myocardial B cells in the naive state to assess the potential of B cell targeting therapies for cardiac diseases. Here, we investigated the ontogeny, trafficking dynamics, histology, and gene expression profiles of myocardial B cells in order to resolve the apparent inconsistencies between current models of B cell recirculation and the observed populations of $\mathrm{B}$ lymphocytes in the naive heart $(2-5,10)$.

\section{Results}

Murine myocardium contains BM-derived and non-BM-derived $B$ cells. The murine naive heart harbors a relatively large population of B lymphocytes $(2,4,10,11)$. However, it is not clear whether myocardial B cells are embryonic-derived (non-BM-derived) or derived from the bone marrow (BM). Since embryonic-derived B lymphocytes accumulate in specific niches within tissues (12), we hypothesized that myocardial B cells would be predominately composed of non-BM-derived cells. To test this hypothesis, we transplanted BM from 
CD45.1 mice into lethally irradiated CD45.2 B cell-deficient animals ( $\mu$ MT) (13). Myocardial B cells can be grouped into 3 subpopulations: $\mathrm{CD} 19^{+} \mathrm{CD} 11 b^{-}, \mathrm{CD} 19^{+} \mathrm{CD} 11 \mathrm{~b}^{+} \mathrm{IgM}^{+} \mathrm{CD}^{+}$, and $\mathrm{CD} 19^{+} \mathrm{CD} 11 \mathrm{~b}^{+} \mathrm{IgM}^{+} \mathrm{CD} 5$ (2). BM transplant reconstituted 2 of $3 \mathrm{~B}$ cell populations in the heart (Figure 1, A and B). Hearts from BM transplant recipients harbored $\mathrm{CD} 19^{+} \mathrm{CD} 11 \mathrm{~b}^{-}$and $\mathrm{CD} 19^{+} \mathrm{CD} 11 \mathrm{~b}^{+} \mathrm{IgM}^{+} \mathrm{CD} 5^{-} \mathrm{B}$ cell populations but lacked $\mathrm{CD}^{2}{ }^{+} \mathrm{CD} 11 \mathrm{~b}^{+} \mathrm{IgM}^{+} \mathrm{CD}^{+}$cells, suggesting that the latter population is not derived from $\mathrm{BM}$ progenitors, thus disproving our initial hypothesis. To confirm these results and to eliminate potential confounding effects associated with radiation-induced damage to the heart, we repeated the experiment by performing limited $\mathrm{BM}$ ablation through focused irradiation of the femurs. This altered irradiation strategy did not qualitatively alter the results and confirmed that most myocardial B cells are BM derived (Supplemental Figure 1, A-C; supplemental material available online with this article; https://doi.org/10.1172/jci.insight.134700DS1).

Myocardial B cells recirculate between the heart, blood, and spleen. The investigation of the origin of myocardial B cells described above did not explain the existence of a large pool of myocardial B cells. Therefore, we formulated a different hypothesis. Since several populations of resident lymphocytes have been described (14), and the heart harbors resident leukocyte populations (15), we hypothesized that myocardial $\mathrm{B}$ cells might represent a population of tissue-resident B lymphocytes. To test this hypothesis, we conjoined CD45.1 and CD45.2 mice via parabiosis and analyzed myocardial B cell chimerism 3 weeks after surgery. The results indicated that both $\mathrm{CD} 19^{+} \mathrm{CD} 11 \mathrm{~b}^{+}$and $\mathrm{CD} 19^{+} \mathrm{CD} 11 \mathrm{~b}^{-}$cells displayed approximately $50 \%$ chimerism after 3 weeks of parabiosis (Figure 1C), suggesting that the vast majority of myocardial B cells have immediate access to the circulation and freely recirculate between conjoined animals. To confirm this result and investigate the trafficking dynamics of myocardial B cells, we performed heterotopic heart transplant. We transplanted the heart of a CD45.2 mouse into the abdomen of a CD45.1 mouse so that both hearts were connected to the vasculature and perfused at the same time. Untransplanted hearts from CD45.1 and CD45.2 mice displayed clear populations of CD45.1 ${ }^{+} \mathrm{B}$ cells and CD $45.2^{+} \mathrm{B}$ cells, respectively (Figure 1D). The transplant recipient mice were sacrificed 4 days after surgery, and the recipient and transplanted hearts were harvested and analyzed. Virtually all donor-derived B cells in the transplanted heart were replaced by recipient-derived B cells (Figure 1D). Only a very small population of donor-derived cells remained in the transplanted heart (Figure 1D). A small population of donor-derived cells was detected in the endogenous heart of the recipient mouse (Figure 1D), whereas the vast majority of cells transplanted with the donor heart were not observed in the myocardial tissue of the recipient mouse. This result confirmed that the vast majority of myocardial B cells are not tissue-resident cells and also suggested that myocardial B cells can migrate from the heart to other organs.

To identify where myocardial B cells traffic after leaving the heart, we analyzed the spleen and blood of mice that received the heart transplant. The results showed that, similarly to the myocardium, the spleen and blood of recipient mice also contained a small but clearly detected fraction of $\mathrm{B}$ cells derived from the donor heart (Figure 2A), suggesting that myocardial B cells recirculate between the heart, blood, and spleen. We confirmed this result by performing adoptive transfer of splenocytes into Rag1 ${ }^{-/-}$mice, which are deficient in B cells and lack myocardial B cells (Figure 2B). Four weeks after adoptive transfer of splenocytes from a WT mouse, Rag1 ${ }^{-/-}$mice had detectable myocardial B cells. The WT spleen is known to harbor both BM-derived and non-BM-derived B cells, and accordingly, adoptive transfer of WT splenocytes reconstituted all 3 subpopulations of myocardial B cells (Figure 2B).

Myocardial B cells are primarily intravascular and closely contact the endothelium. Our data failed to explain why a relatively large population of B cells is observed in perfused naive hearts. Therefore, we investigated the histological location of myocardial B cells, specifically examining whether they were intravascular or intraparenchymal, by generating a CD19-tdTomato reporter mouse that efficiently labeled B cells (Supplemental Figure 1, D-F). The results showed that myocardial B cells were sparsely distributed throughout the myocardium (Figure 3A). When we stained for CD31 sections from CD19-tdTomato reporter animals to identify the vasculature and localize B cells in relationship to the endothelium, we found that most myocardial B cells were intravascular. Most B cells were in the microvasculature and were in close proximity to surrounding endothelial cells (Figure 3B). Some B cells were found in clusters of 2 or 3 cells (Figure 3C). Few B cells were observed in the intraparenchymal/extravascular space; these were isolated cells or small clusters of 2-3 cells (Figure 3D, Supplemental Figure 1E, and Supplemental Video 1). Rare B cells were found to be in transit through the endothelium (Figure $3 \mathrm{E}$ and Supplemental Video 2). To confirm the intravascular localization of myocardial B cells, we performed intravascular injection of a CD45.2 antibody immediately before 
A

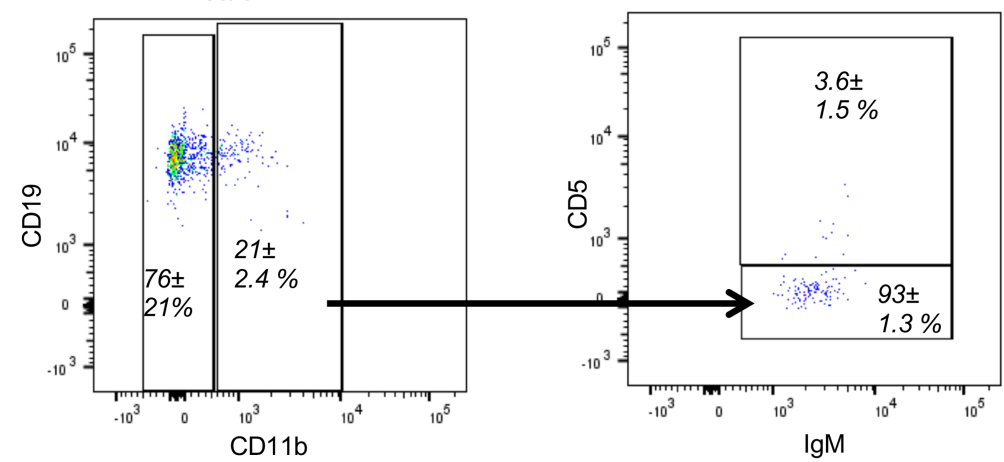

C

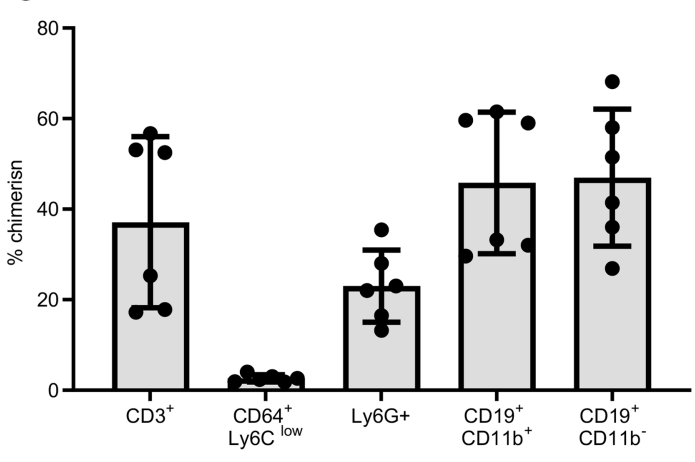

B $\quad \mu M T$ heart after BMT

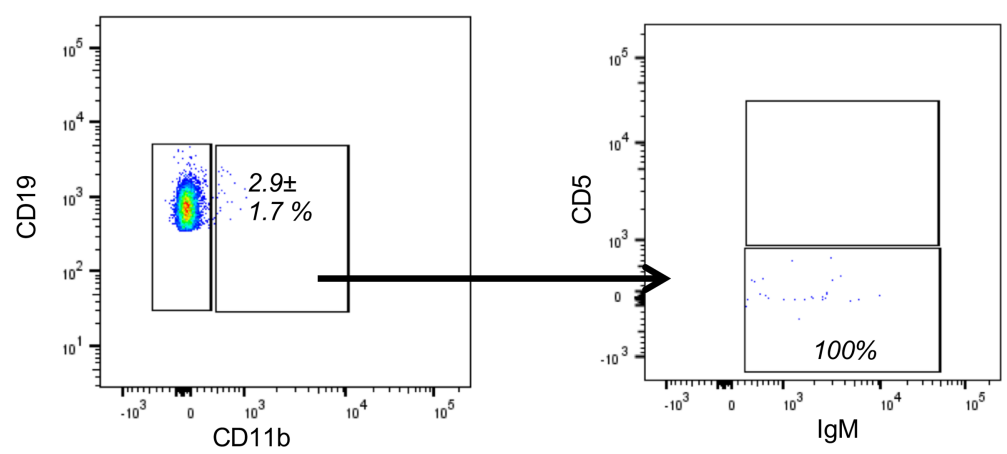

D

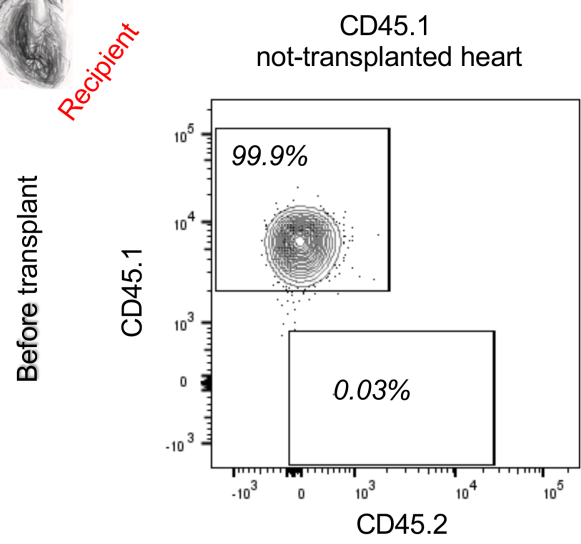

CD45.1

Transplant recipient heart

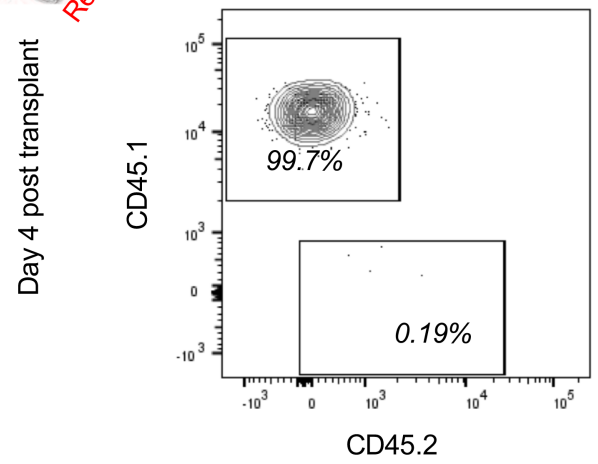

CD45.2

not-transplanted heart

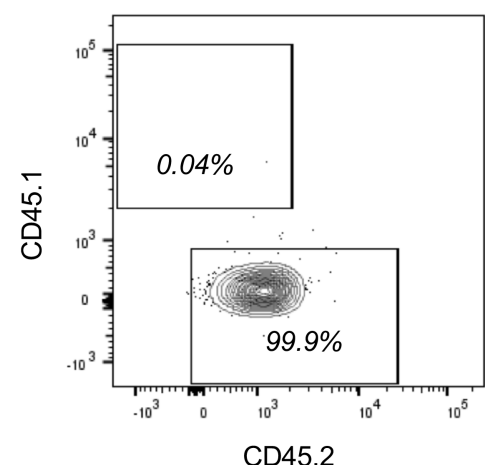

CD45.2

transplanted heart

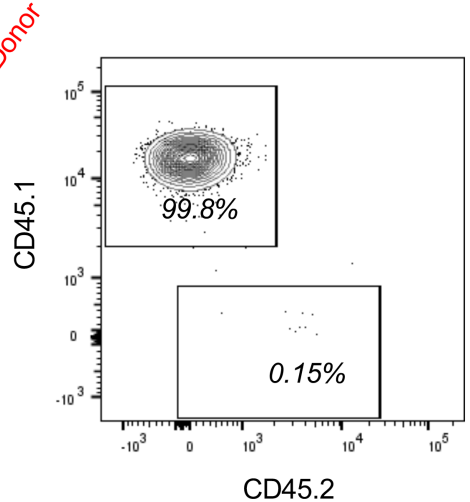


Figure 1. Myocardial B cells have disparate origin and, for the most part, are not resident cells. (A) Flow cytometric analysis of $C D 45^{+} C D 19^{+}$myocardial cells in a [57/B6] mouse. Most myocardial B cells are CD11b-. Among the CD19+CD11b+ myocardial B cells, the majority are IgM+CD5-, and a small portion are $\operatorname{lgM}{ }^{+} \mathrm{CDS}^{+}$. The flow cytometry plots are representative of 3 animals analyzed in different experiments. Mean percentage of total CD19+ cells \pm SD is reported next to each gate. (B) Flow cytometric analysis of CD45+CD19+ myocardial cells in [57/B6] B cell-deficient mice ( $\mu$ MT) after BM transplant with WT BM. BM transplant replenished CD11b cells more efficiently than CD11b+ cells. In the CD11b ${ }^{+}$compartment, BM transplant did not produce IgM+CD5+ cells. The flow cytometry plots are representative of 4 animals analyzed in different experiments. Mean percentage of total CD19+ cells \pm SD is reported

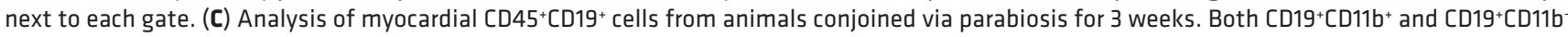
cells showed $50 \%$ chimerism, a finding consistent with the observation that myocardial B cells moved freely between animals. Percent chimerism for Ly6G+ neutrophils, $C D 64^{+} \mathrm{Ly}^{+} \mathrm{C}^{10}$ macrophages, and $\mathrm{CD}^{+} \mathrm{T}$ cells is shown for comparison. (D) Analysis of $\mathrm{CD} 45^{+} \mathrm{CD} 19^{+}$myocardial $\mathrm{B}$ cells in recipient and donor hearts before transplant and on day 4 after heterotopic heart transplant. Before transplant, the heart of the CD45.1 recipient animal contains only CD45.1+ B cells, and the donor heart from a CD45.2 animal contains only CD45.2+ B cells. Four days after transplant, the recipient heart is mostly unchanged, though it contains a small population of $C D 45.2^{+} B$ cells derived from the transplanted heart (left side). The CD45.2 transplanted heart instead has lost almost all of its CD45.2+ B cells and now contains mostly CD45.1+ recipient-derived B cells. Representative flow cytometry plots from 3 independently transplanted animals. Percentage of total $\mathrm{CD} 19^{+}$cells is reported within each gate.

sacrificing the mice and collecting the hearts for flow cytometric analysis. Under these conditions, only cells located in the intravascular space are stained by the injected antibody (16). Consistent with our histological analysis, essentially all myocardial B cells were stained by the CD45.2 antibody (Figure $3 \mathrm{~F})$. Only $3 \%$ of myocardial B cells were not stained by the CD 45.2 antibody, indicating that they were not in the intravascular space at the time of analysis (Figure 3F, bar graph).

Myocardial B cell transcriptional profiles differ from those of circulating $B$ cells. Our results did not exclude the possibility that myocardial B cells might simply represent circulating B cells that were left in the myocardial vasculature at the time of organ harvesting. To address this question, we first compared our standard organ perfusion protocol with large-volume retrograde perfusion after aortic cannulation and confirmed that even the most stringent perfusion protocol did not eliminate myocardial B cells. (Supplemental Figure 2). Then, we performed single cell sequencing of FACS-sorted myocardial B cells and B cells sorted from circulating blood using the Chromium kit and instrument from 10x Genomics (accession number GSE142515). The myocardial and circulating B cell samples were collected from the same mice and at the same time. The results showed that the myocardial B cell transcriptional profile distinctly differed from that of circulating B cells (Figure 4A). We identified the top 5 genes with the highest differential expression levels and frequencies between myocardial and circulating B cells (Supplemental Table 1). Among these 5 genes, we identified 2 that had either available antibodies for flow cytometry (CD83) or an available reporter strain (Nr4al). Flow cytometry analysis showed that myocardial B cells had higher CD83 and NR4a1 expression levels than circulating B cells (Supplemental Figure 2), which confirmed the gene expression analyses. Next, we performed gene set enrichment analysis (GSEA) (17) of the differentially expressed genes in heart and blood B cells. Table 1 shows that myocardial B cells displayed activation of the MAPK signaling pathway, B cell receptor signaling pathway, antigen processing and presentation, chemokine signaling pathway, and leukocyte transendothelial migration.

Myocardial $B$ cells are a subset of circulating $B$ lymphocytes that accumulate in the myocardium. We performed unsupervised clustering of the single-cell transcription data. Virtually all identified clusters were represented in both circulating and myocardial $\mathrm{B}$ cells, which is consistent with the point of view that myocardial B cells recirculate through the blood. However, analyses of the contributions of myocardial and circulating B cells to each cluster indicated that their prevalence differed according to the cluster. Cluster 0 almost exclusively contained myocardial B cells, whereas clusters 1, 2, and 3 almost exclusively contained circulating B cells (Figure 4B). These results suggest that myocardial B cells represent a subset of circulating B lymphocytes that make close contact with the microvascular endothelium in the heart and then arrest their transit. We tested this hypothesis by performing an ex vivo perfusion experiment. We reasoned that, if a subset of circulating $B$ cells adheres to the myocardial vasculature, then perfusing the heart of a mouse deficient in B cells with WT blood would replenish myocardial B cells. We modified a Langendorff perfusion system to perfuse hearts collected from $\mu \mathrm{MT} / \mathrm{CD} 45.2$ B cell-deficient mice with peripheral blood collected from WT CD45.1 mice (Supplemental Video 3). As predicted, perfusion of B cell-deficient hearts with WT blood resulted in repopulation of myocardial B cells similar to that observed in WT hearts (Figure 4C). Next, we confirmed this result by transplanting a WT heart into a syngeneic CD19-tdTomato reporter mouse and performed intravital microscopy. The results showed that, although many circulating B cells rapidly transit through the myocardial vasculature, a significant number of these cells either pause during their transit through the heart or markedly slow down during transit. We observed some B cells moving along the vascular wall with a motion similar to the patrolling movement classically described for monocytes (Figure 4D and Supplemental Video 4) (18). 
A

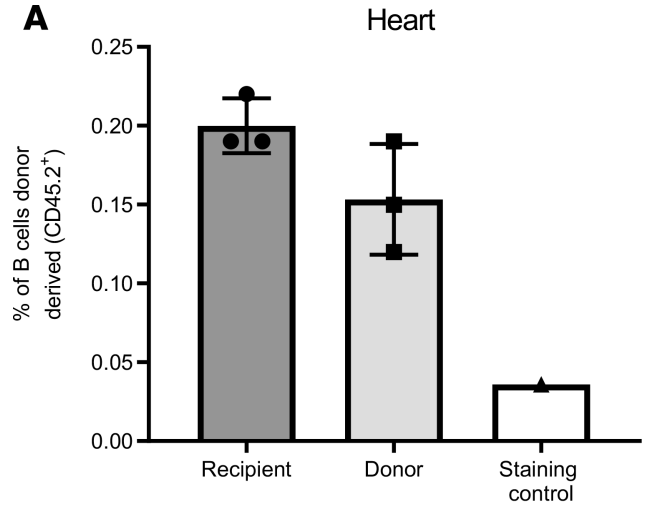

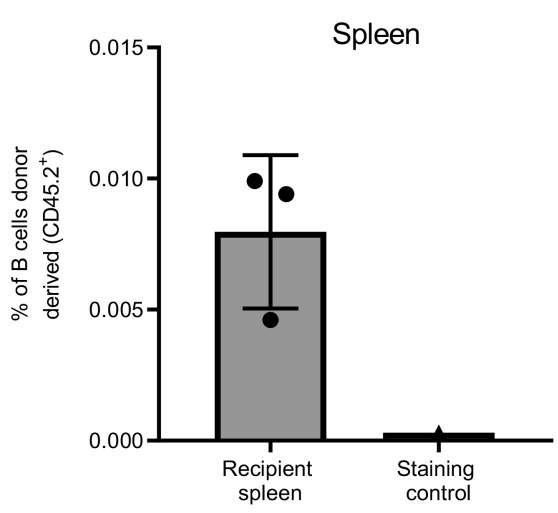

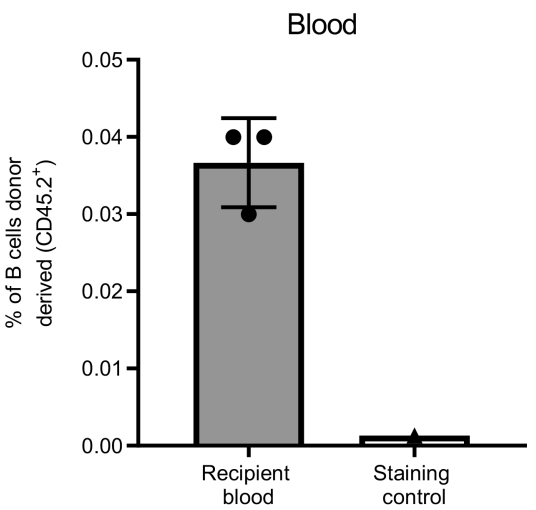

B
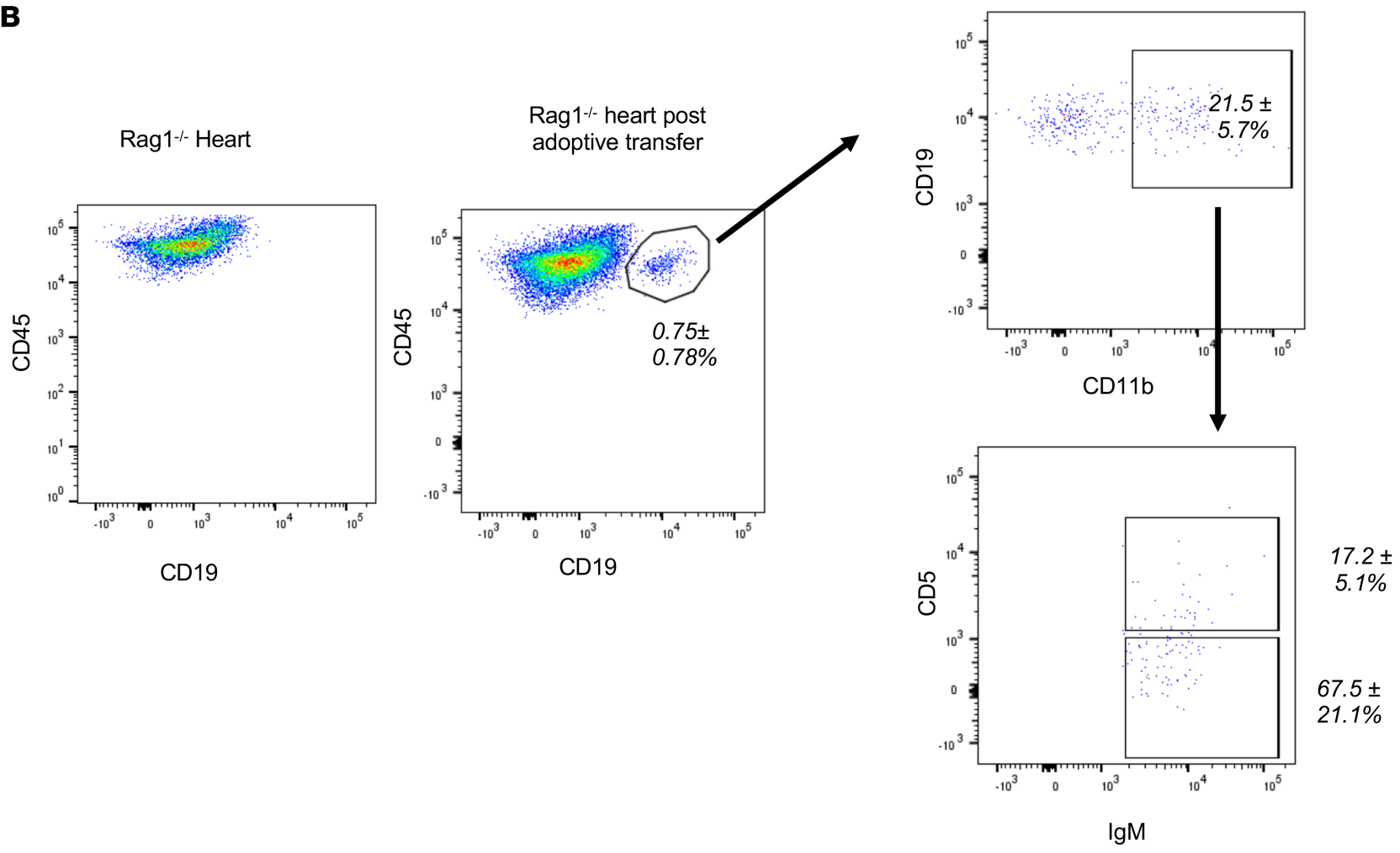

Figure 2. Myocardial B cells recirculate between the heart, the blood, the spleen, and likely other organs. (A) Myocardial, splenic, and circulating (blood) $B$ cells were analyzed 4 days after heterotopic heart transplant, and the percentage of CD45+CD19+ cells that were also CD45.2+ (donor-derived B cell) was calculated. Graph bars represents the percentage of donor-derived B cells found in the recipient heart and in the donor heart (left), recipient spleen (middle), and recipient peripheral blood (right). The percentage of CD45.2+ cells in the absence of the specific antibody (staining control) is reported for reference. Data are expressed as mean \pm SD. (B) A Rag1 ${ }^{-/-}$B cell-deficient mouse was injected with splenocytes from a WT mouse. Three weeks after adoptive transfer, the Rag1 ${ }^{-/-}$mouse has a detectable population of myocardial B cells. Adoptive transfer of splenocytes reconstituted all the 3 major subgroups of myocardial B cells, CD19+CD11 $\mathrm{b}^{-}, \mathrm{CD}_{19}{ }^{+} \mathrm{CD} 11 \mathrm{~b}^{+} \mid \mathrm{IM}^{+} \mathrm{CD} 5^{-}$, and $\mathrm{CD} 19^{+} \mathrm{CD} 11 \mathrm{~b}^{+} \mathrm{IgM} \mathrm{M}^{+} \mathrm{CD} 5^{+}$. The flow cytometry plots are representative of data collected from 5 different animals subjected to adoptive transfer for total myocardial B cells and from 3 different animals for the subgroup analysis of myocardial B cells. Average percentage of total $C D 45^{+}$cells is reported in the CD45/CD19 plot, while average percentage of the parent gate is reported in the other plots. Mean $\pm \mathrm{SD}$ is reported in each plot next to each average value.

Myocardial B cells are primarily naive follicular $B$ cells. To further identify specific subtypes of myocardial B cells, we analyzed the single-cell gene expression data. Myocardial B cells expressed Stat4, Nkbb2, Rel-a, Rel-b, Klf-3, and Klf-6 but did not express Blmp1, Tox, Pbx3, Klf-9, or Ahr (Supplemental Figure2C). This gene expression profile is consistent with that of naive B cells $(19,20)$. Nr4a1 was the most strongly upregulated gene in the differential gene expression analysis (Supplemental Table 1) and has been implicated in downstream signaling of the B cell receptor (BCR) (21). To address whether activation of BCR signaling was responsible for B cell adherence to the endothelium, we compared the number of myocardial B cells between genetically modified mice that uniformly express a $\mathrm{BCR}$ with specificity for a nonmurine antigen 
A

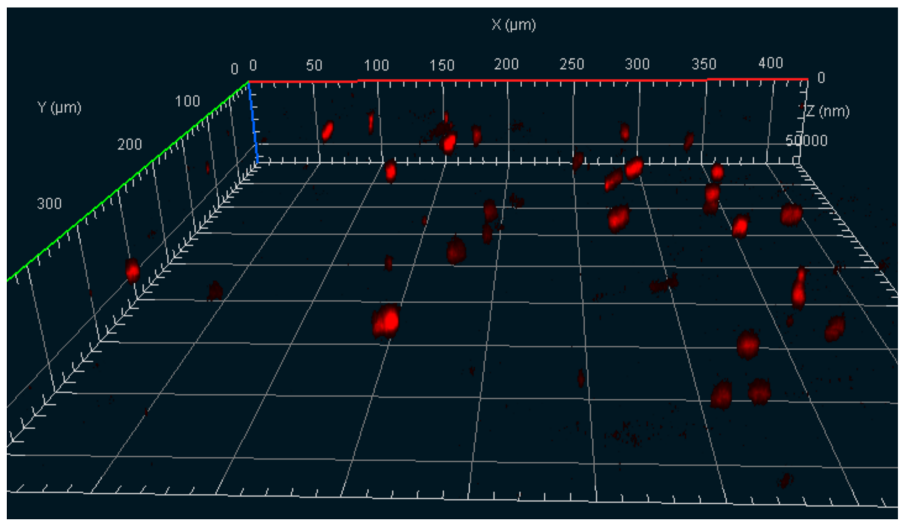

C Most $\mathrm{B}$ cells are in capillaries

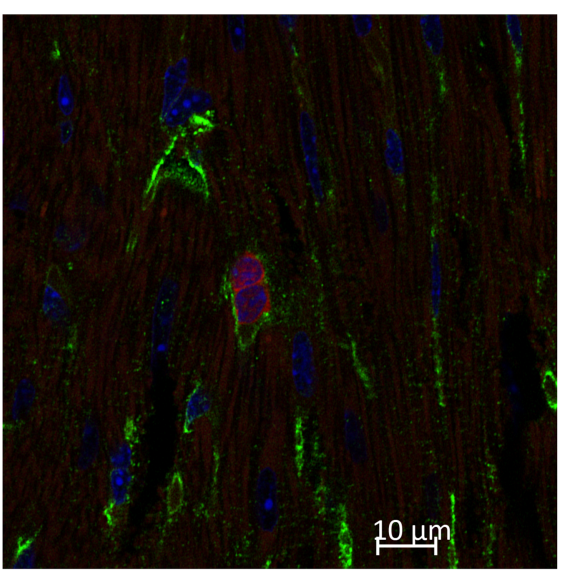

D

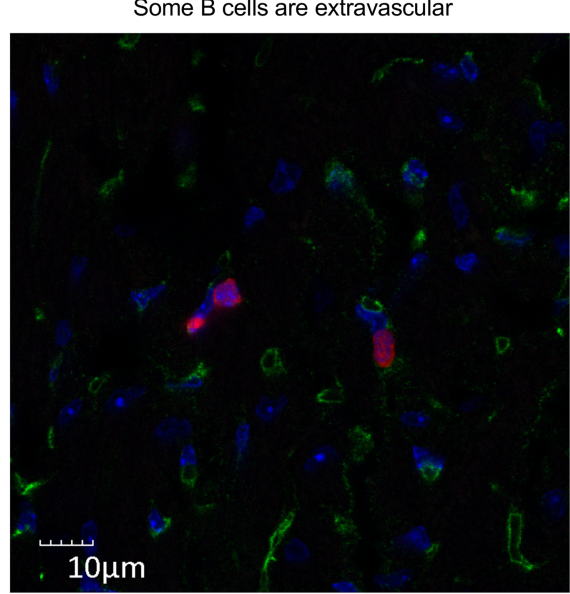

B

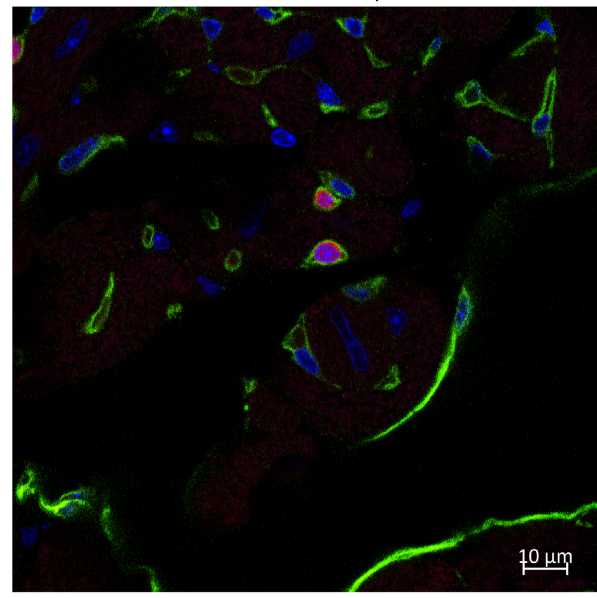

E Rare B cells are transiting through the

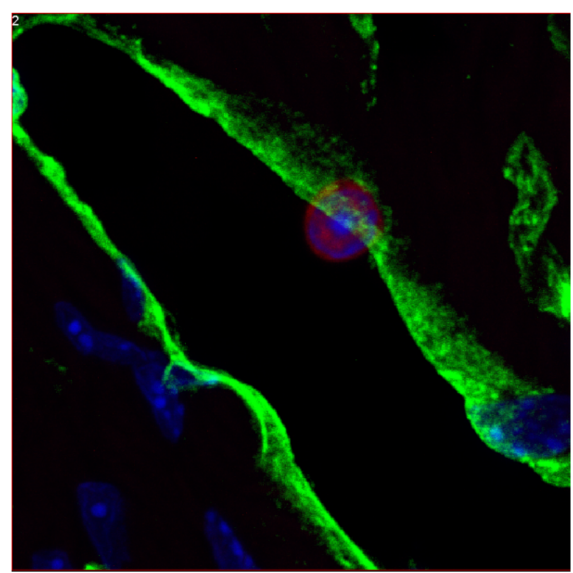

$\mathbf{F}$

Myocardial CD19+ cells
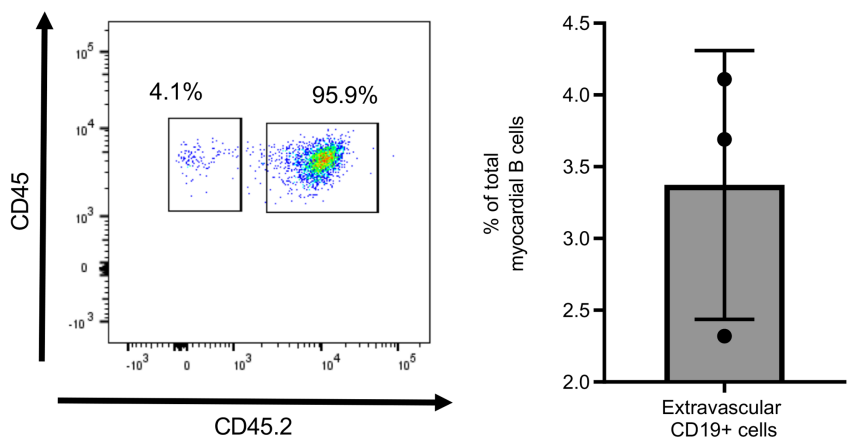

Figure 3. Myocardial B cells are mostly intravascular and in intimate contact with the endothelium. (A) Confocal imaging of a section of myocardial tissue from a CD19-tdTomato reporter animal. The 3-dimensional reconstruction of multiple images acquired along the $z$ axes shows that $B$ cells are distributed throughout the myocardium. LSM 880 Indimo, AxioObserver Zeiss Microscope using a Plan-Apochromat $10 \times / 0.8$ M27 objective. Z-stack of 69 slices (64.829 uM). (B-E) Confocal images of a frozen section of murine myocardium. CD19-tdTomato, red; CD31, green; DAPI, blue. Myocardial B cells are mostly intravascular and in intimate contact with the endothelium. Taken with an LSM 880 Indimo, AxioObserver Zeiss Microscope using a Plan-Apochromat 63×/1.3 oil DIC UV-IR M27 objective and 2x digital zoom (B). Some B cells are in pairs. Taken with an LSM 880 Indimo, AxioObserver Zeiss Microscope using a Plan-Apochromat 40×/1.3 oil DIC UV-IR M27 objective and a 2.2 digital zoom (C). Few B cells are found in the intraparenchymal/extravascular space, as singlets or doublets. taken with an Olympus Fluoview FV1000 using a PLAP0N 60× NA1.4 objective and $2 \times$ digital zoom (D); rare B cells are found in transit through the endothelium, taken with the same microscope and objective as $\mathbf{D}$ but it is a Z-stack of 23 slices, $0.46 \mathrm{uM} /$ slice (E). (F) Flow cytometric analysis of myocardial CD19+CD45 ${ }^{+}$cells 3 minutes after i.v. injection of a CD45.2 antibody. Most myocardial B cells are stained by the i.v. injected antibody, confirming their intravascular location. Only about $3 \%$ of cells are not stained by the antibody and are, therefore, extravascular. The flow cytometry plot is representative of 3 independent experiments. Percentage of total CD19+ cells is reported next to each gate. The bar graph reports mean percentage of extravascular CD19+ cells \pm SD. 
A

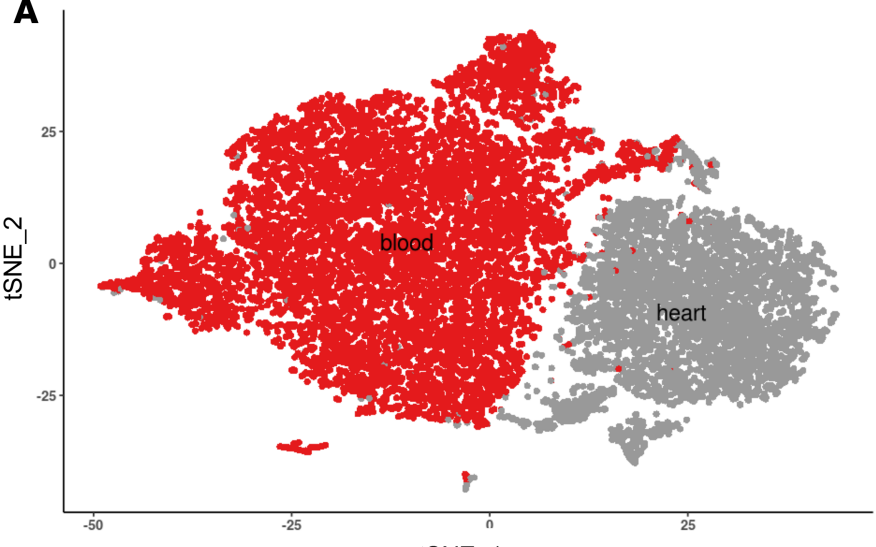

D

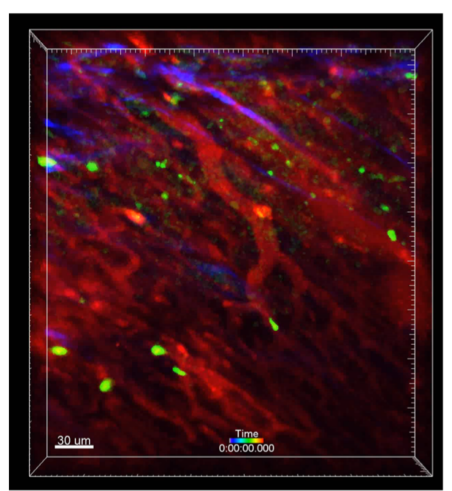

tSNE_1

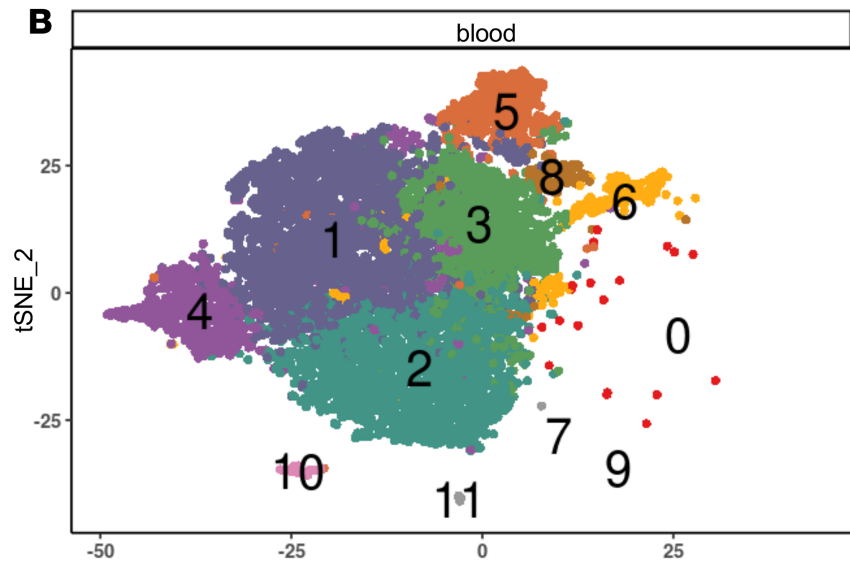

- blood

- heart

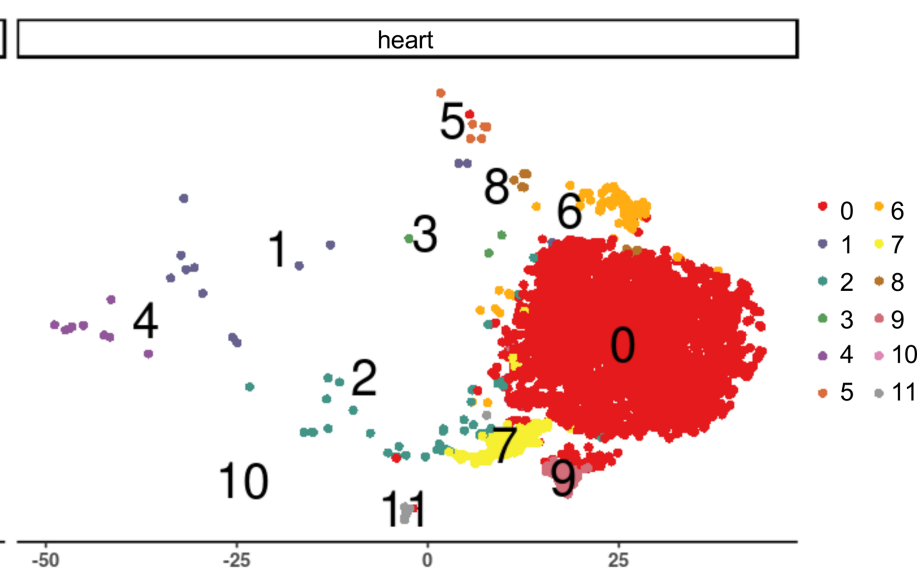

tSNE_1

C

CD45-1 ${ }^{+}$, Live/Dead Aqua

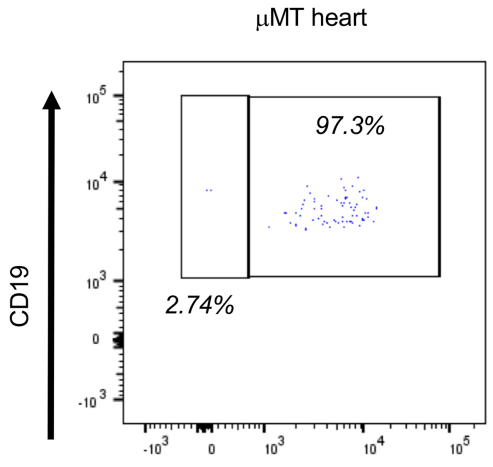

$\mu \mathrm{MT}$ heart perfused with blood and flushed
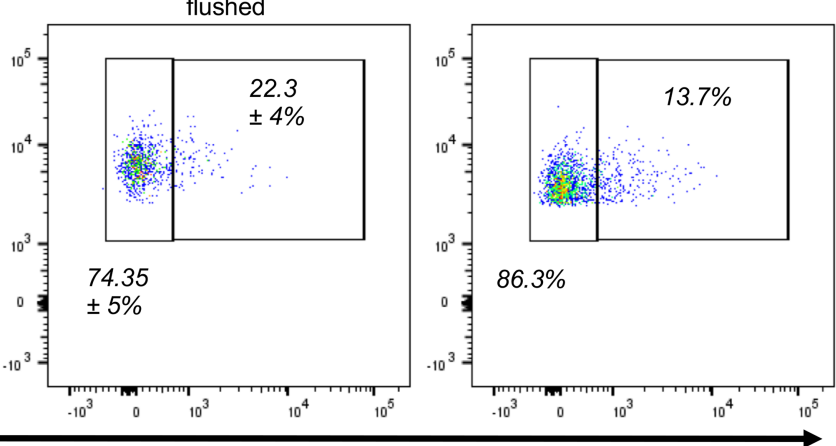

CD11b

Figure 4. Myocardial B cells are a subset of circulating B lymphocytes with distinct transcriptome that adheres to the myocardial endothelium. (A) tSNE plot of 10x single cell sequencing data from CD45+CD19+ cells sorted from the heart and the blood of the same pool of WT 10-week-old C57/B6] mice. B cells sorted from the heart have a different gene expression profile than B cells sorted from the blood. The experiment was repeated twice. (B) tSNE plot integrated with unsupervised clustering. Myocardial (heart) and circulating (blood) B cells overall contain cells with similar gene expression profile. However, specific clusters of cells, such as cluster 0 , are enriched in the heart, while other clusters of cells, such as cluster 1 , are enriched in the blood. (C) The heart from a $\mu \mathrm{MT}$ mouse has almost no myocardial B cells (panel on the left, representative plot). After ex vivo perfusion with WT blood and rinsing, the $\mu$ MT heart contains a sizeable population of CD19+ $B$ cells (panel in the middle, $n=3$ reporting average percentage of cells in each gate \pm SD), which is very similar to that observed in the heart of a WT animal (panel on the right). Representative FACS plots. (D) Still image from intravital microscopy of B cells flowing through a transplanted heart. B cells are depicted in green, while the vasculature is depicted in red. A number of B lymphocytes is still and well visible within the myocardial microvasculature (see Supplemental Video 4). Representative image from videos collected from multiple hearts. 
Table 1. Pathway analysis of genes differentially expressed between myocardial and circulating B cells

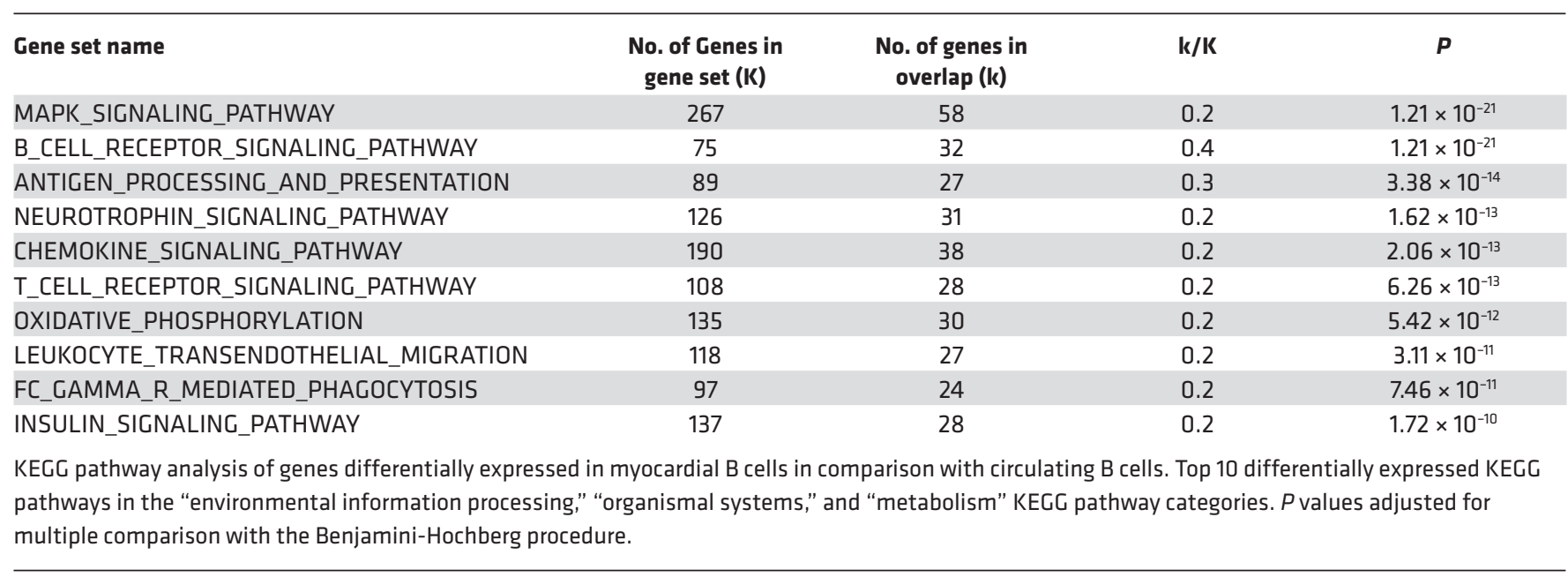

(HEL mice) and syngeneic, age/sex-matched WT control mice. The results showed that HEL mice did not have markedly fewer myocardial B cells than control mice, suggesting that BCR specificity is not a major driver of B cell accumulation in the heart (Supplemental Figure 4A).

$B$ cell deficiency alters the myocardial leukocyte pool, reduces myocardial mass, and alters left ventricular contractility. Next, we investigated the functional significance of B lymphocytes in the naive heart by comparatively analyzing the myocardium of WT mice and that of syngeneic, age/sex-matched B cell-deficient $(\mu \mathrm{MT})$ mice. We first analyzed the myocardial leukocyte pool using flow cytometry; Supplemental Figure 4B describes the gating strategy. The results showed that B cell-deficient mice had fewer myocardial Ly $6 \mathrm{C}^{+}$monocytes and more $\mathrm{CD}^{+}$and $\mathrm{CD}^{+}$myocardial $\mathrm{T}$ cells (Figure $5 \mathrm{~A}$ ). We also investigated the role of induced versus genetic $\mathrm{B}$ cell depletion with respect to modulating the myocardial leukocyte pool. WT mice were administered anti-CD20 antibody (2) to deplete their B cells; then, they were compared with isotype control-treated mice. Antibody-mediated B cell depletion numerically increased $\mathrm{Ly}_{6 \mathrm{G}}{ }^{+}$neutrophils and significantly increased myocardial $\mathrm{CD} 4^{+}$ and $\mathrm{CD} 8^{+} \mathrm{T}$ cells (Figure 5B). Although the effect of chronic genetic B cell depletion on the myocardial leucocyte pool differed from that of acute antibody-mediated B cell depletion, the results of both experiments support the hypothesis that B cells modulate the leukocyte pool in the naive heart.

We examined myocardial structure and left ventricular systolic function in $\mu \mathrm{MT}$ B cell-deficient mice and matched syngeneic WT controls. We analyzed myocardial structure by assessing cardiomyocyte and myocardial size via wheat germ agglutinin (WGA) staining and gravimetric analysis, respectively. The $\mu \mathrm{MT}$ mice had a smaller myocardial fiber cross-sectional area and lower myocardial mass than age-matched syngeneic controls (Figure 5, C and D). Left ventricular function was assessed via echocardiography. The $\mu \mathrm{MT}$ $\mathrm{B}$ cell-deficient mice had higher left ventricular ejection fraction and faster left ventricular relaxation than matched syngeneic controls (Figure 5E, Supplemental Table 2, and Supplemental Figure 5).

Human heart harbors intravascular B cells in close association with the endothelium. We explored the relevance of our results to humans by analyzing fresh specimens of human left ventricle collected at the time of left ventricular assist device implantation. Human heart tissue was enzymatically digested and analyzed via flow cytometry. The results showed that human heart harbored a population of B cells (Figure 5F). Next, we stained histological sections of human myocardium with an antibody recognizing the B cell-specific antigen CD20. We observed that human myocardial B cells were almost exclusively intravascular and in close association with the endothelium (Figure 5G), similar to what we observed for murine myocardial B cells.

\section{Discussion}

Gowans and Knight performed the classic studies of B lymphocyte recirculation in the 1960s (8). Since then, immunologists have believed that B lymphocytes continuously recirculate between primary and secondary lymphoid organs without accumulating in peripheral tissues (7-9). However, we and others have observed a relatively large pool of $\mathrm{B}$ lymphocytes in the naive heart $(1-5,10)$. The present study investigated this 

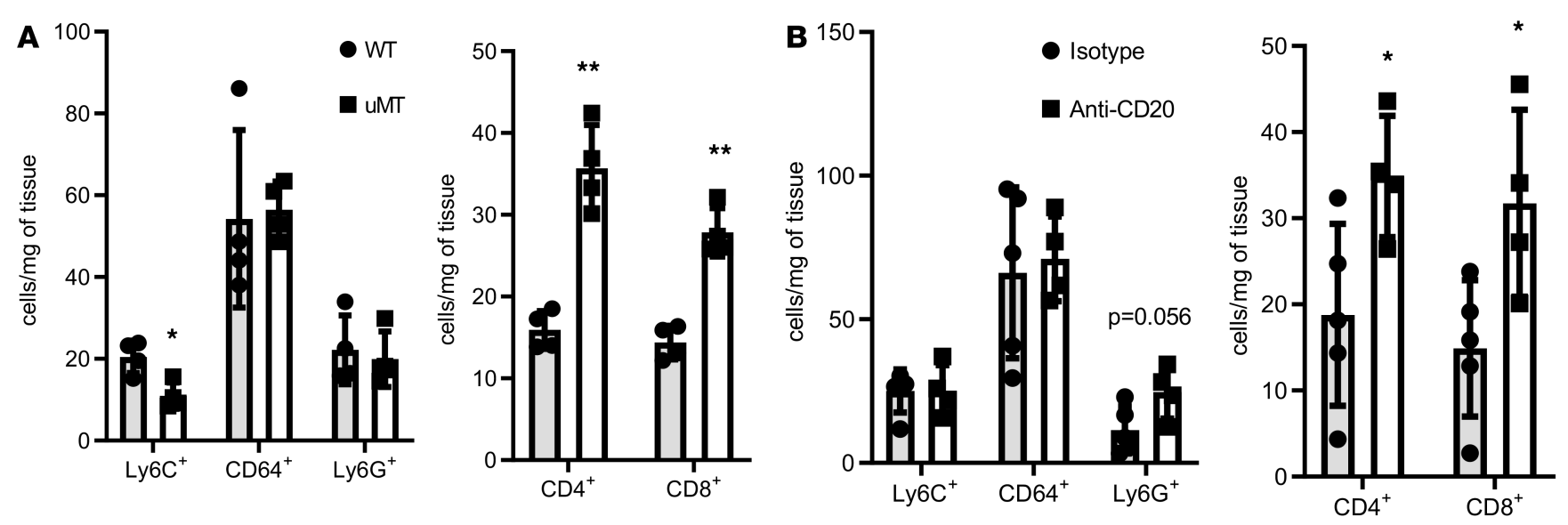

C

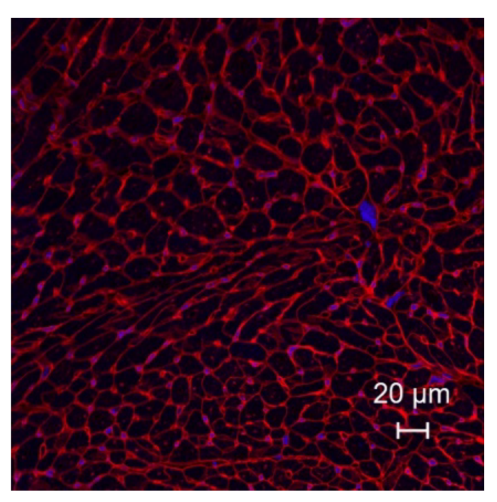

D

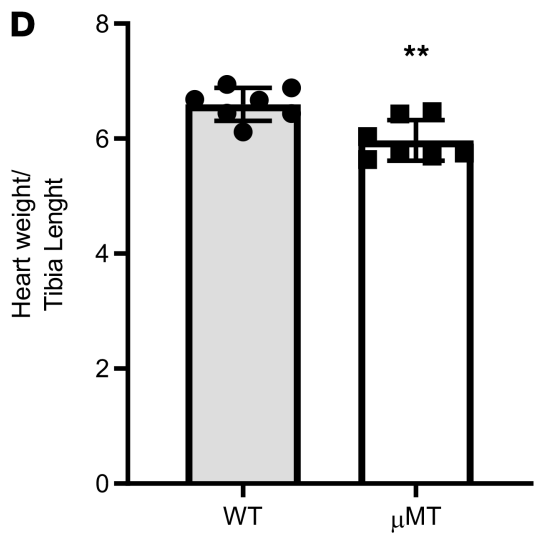

F CD45+, Live/Dead Aqua - cells

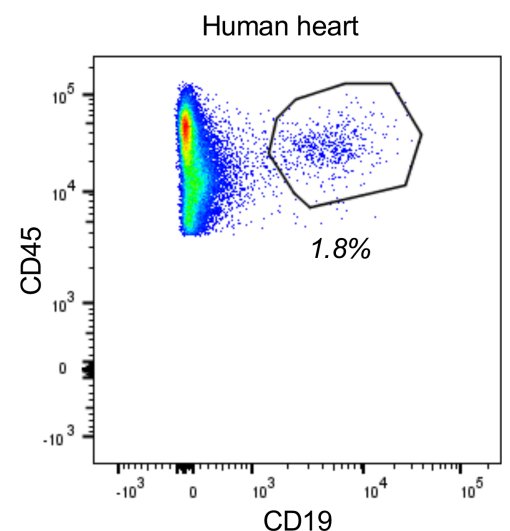

$\mathbf{E}$

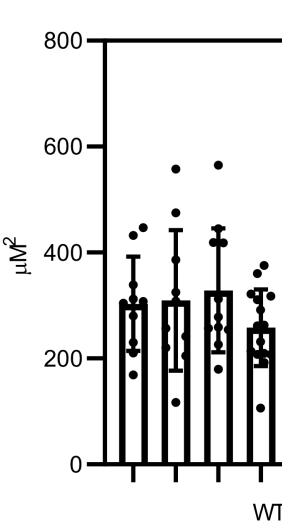

$p=0.036$

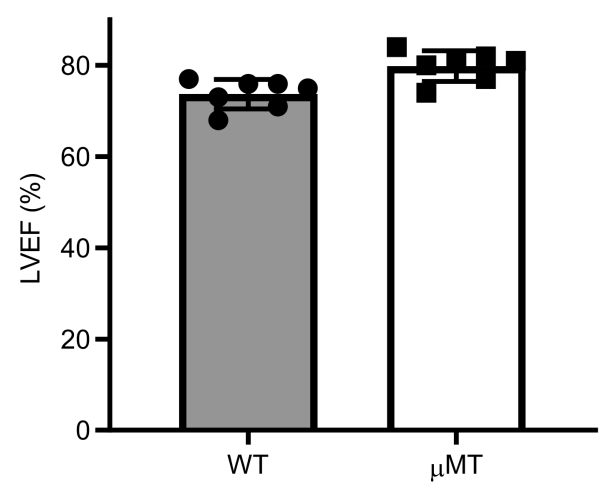

G Endothelial cell

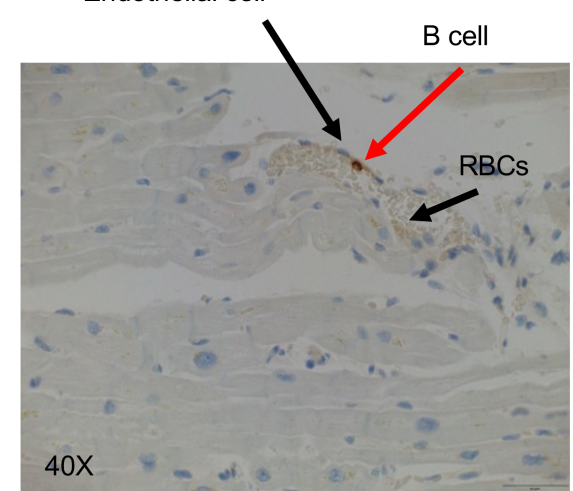

$\mu \mathrm{MT}$

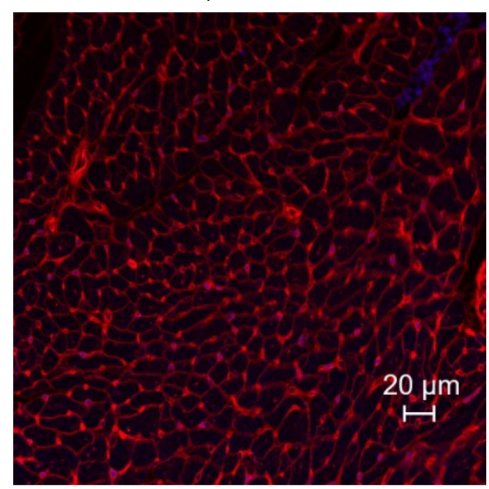

$\mathrm{dV} / \mathrm{dT}$
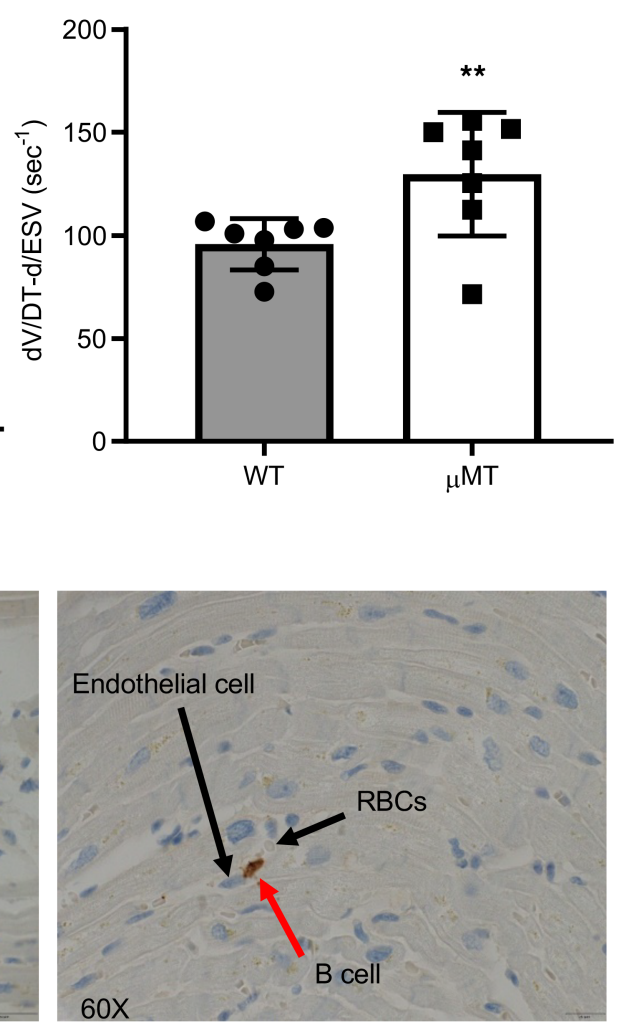
Figure 5. B cell deficiency alters the myocardial leukocyte pool, reduces myocardial mass, and alters left ventricular contractility. (A) Flow cytometry analysis of myocardial leucocytes in WT and $\mu \mathrm{MT}$ mice. $\mu \mathrm{MT}$ mice have less $\mathrm{Ly}_{6 \mathrm{C}}^{+}$cells and more $\mathrm{CD}^{+}{ }^{+}$and $\mathrm{CD} 8^{+} \mathrm{T}$ cells. (B). Flow cytometric analysis of WT animals depleted of B cell through administration of anti-CD20 antibody or isotype control. Antibody-mediated B cell depletion is associated with an increase in $\mathrm{Ly6G}^{+}$cells $(P=0.056), \mathrm{CD} 4^{+}$and $\mathrm{CD} 8^{+}$cells. (C) Analysis of hearts from 27-week-old $\mathrm{C57/B6)}$ mice and syngeneic age/sex-matched $\mu$ MT mice showed that $\mu$ MT mice have myocardial fibers with smaller cross-sectional area as assessed by WCA staining. Representative images of WGA staining for each group are shown and cumulative data are reported. (D) $\mu \mathrm{MT}$ mice have lower myocardial mass as assessed by heart weight/tibia length $(\mathrm{mg} / \mathrm{mm})$. (E) Echocardiographic analysis of left ventricular function in WT mice and matched $\mu$ MT mice. $\mu$ MT mice have higher left ventricular ejection fraction (LVEF) and faster ventricular relaxation (dV/dT-d, volume change per unit of time during diastolic relaxation; ESV, end-systolic volume). (F) Flow cytometry analysis of a sample of human myocardium collected at the time of implantation of a left ventricular assist device and dissociated enzymatically. A clear population of $\mathrm{CD} 19^{+}$cells is shown. Representative plot from 3 heart samples analyzed. Percentage of myocardial CD45+ cells is reported next to the gate. (G) Histological analysis of human myocardium collected at the time of LVAD placement shows that, similarly to the murine heart, the human heart harbors B cells in the intravascular space, in intimate contact with the endothelium. Magnification, 40x. Pairwise comparisons were performed with 2-tailed $t$ test. Data are expressed as average \pm SD. The WT and $\mu$ MT groups were compared using unpaired 2-tailed $t$ test adjusted for multiple comparisons with the FDR method in $\mathbf{A}$ and $\mathbf{B}$. In $\mathbf{C}$, the 2 groups were compared using nested $t$ test ${ }^{*} P<0.05$. ${ }^{* *} P<0.01$.

apparent inconsistency and found that a subpopulation of circulating B cells makes close contact with the microvascular endothelium in the heart and arrest their transit as they pass through the heart. These myocardial B cells upregulated specific signaling pathways, such as antigen processing and presentation and chemokine-chemokine signaling, and patrolled along the endothelium. These results redefine the current paradigm of B lymphocyte recirculation in the naive state. In addition, we observed that B lymphocytes modulated the myocardial leukocyte pool composition, myocardial mass, and left ventricular contractility in B celldeficient or -depleted mice. Our observations revealed an unexpected relationship between circulating B lymphocytes and myocardial biology, thereby challenging the widespread belief that leukocytes function in tissue homeostasis only after extravasating and entering the parenchyma (16).

We began our investigation of $\mathrm{B}$ cells in the naive heart by identifying the origin of the 3 murine myocardial B cell subpopulations: $\mathrm{CD} 19^{+} \mathrm{CD} 11 \mathrm{~b}^{-}, \mathrm{CD} 19^{+} \mathrm{CD} 11 \mathrm{~b}^{+} \mathrm{IgM}^{+} \mathrm{CD} 5^{+}$, and $\mathrm{CD} 19^{+} \mathrm{CD} 11 \mathrm{~b}^{+} \mathrm{IgM}^{+} \mathrm{CD} 5^{-}$. We observed that $\mathrm{BM}$ transplant effectively repleted the $\mathrm{CD} 19^{+} \mathrm{CD} 11 \mathrm{~b}^{-}$population, repleted the $\mathrm{CD} 19^{+} \mathrm{CD}$ $11 \mathrm{~b}^{+} \mathrm{IgM}^{+} \mathrm{CD} 5^{-}$population with low efficiency, and failed to replete the $\mathrm{CD} 19^{+} \mathrm{CD} 11 \mathrm{~b}^{+} \mathrm{IgM}^{+} \mathrm{CD} 5^{+}$population (Figure 1, A and B). Based on the existent literature (22), our findings suggest that myocardial $\mathrm{CD} 19^{+} \mathrm{CD}$ $11 \mathrm{~b}^{+} \mathrm{IgM}^{+} \mathrm{CD}^{+}$cells are embryo-derived B1a cells, whereas $\mathrm{CD} 19^{+} \mathrm{CD} 11 \mathrm{~b}^{+} \mathrm{IgM}^{+} \mathrm{CD}^{-}$and $\mathrm{CD} 19^{+} \mathrm{CD} 11 \mathrm{~b}$ myocardial B cells are, respectively, B1b BM- and B2 BM-derived cells. These observations suggest that the murine myocardium harbors B cells with different ontogenies.

We studied the dynamics of myocardial B cell trafficking using parabiosis and heart transplant. The analysis of parabiotically conjoined mice indicated that both B1 and B2 myocardial B cells undergo recirculation (Figure 1C). Analyses of mice subjected to heterotopic heart transplant confirmed this result and suggested that the rate of B cell transit through the heart is relatively fast, as only $0.2 \%$ of donor-derived myocardial B cells were observed in the transplanted heart at 4 days after surgery (Figure 1C). Analyses of blood, heart, and spleen from the heart transplant recipient mice suggested that myocardial B cells likely migrate widely within murine tissues (Figure 2). Our experimental design did not enable quantification of the number of $B$ cells transplanted with each heart or of the number of B cells recovered in different organs analyzed at the time of harvest. Therefore, we could not determine whether some of the transplanted myocardial B cells died within the transplanted heart or migrated to other recipient organs that were not analyzed.

Given that we consistently observed relatively large pools of myocardial B cells in flow cytometric analysis of digested hearts, we performed a histological analysis of myocardial B cells to define their anatomic location and their relationship with the endothelium. The results showed that $\mathrm{B}$ cells were almost exclusively intravascular in naive perfused hearts (Figure 3). One possibility is that these myocardial B cells may be an artifact of poor perfusion at the time of tissue harvesting. However, extensive perfusion during repeated experiments reduced but did not eliminate the observed myocardial B cell pools (Supplemental Figure 2), and single cell sequencing indicated that myocardial B cells have distinct gene expression patterns when compared with circulating B cells (Figure 4A). Clustering analysis showed that myocardial B cells are highly enriched in specific naive circulating B cell subtypes (cluster 0 in Figure 4C). These results should be interpreted with caution because of the small number of events recorded within cluster 0 in the sample of circulating B cells. However, since myocardial B cells are predominately intravascular, these combined results suggested that subtypes of circulating B cells with specific properties adhere to the endothelium and have longer permanence time in the myocardium than other subtypes. The observation that some circulating B cells adhere to the endothelium and persist in the myocardium was confirmed by our ex vivo perfusion study (Figure 4D) and intravital microscopy imaging (Figure 4E and 
Supplemental Video 4). Although neither of these experiments analyzed completely naive hearts, both experiments exposed naive hearts to cold ischemia, which was minimized to $<10$ minutes for the ex vivo perfusion experiments and to $<30$ minutes for the intravital microscopy experiments. Therefore, we consider that it is reasonable to conclude that the observed results are relevant to naive heart biology.

Circulating B cells are primarily naive follicular B cells (23), and the gene expression profiles of myocardial B cells across gene expression clusters were consistent with this B cell subtype (Supplemental Figure 3). This result suggests that myocardial B cells are primarily a subgroup of naive follicular B cells that transitioned into a specific functional state. Our data suggest that myocardial B cells are naive circulating B cells that are prone to engaging the endothelium and upregulate patrolling and antigen processing functions in the context of immune surveillance. Further studies will be needed to verify this hypothesis and to understand whether these "slow transiting" B cells that we have observed in the heart are specific to the myocardium or exist also in other organs.

The myocardial leukocyte pool compositions of B cell-deficient and B cell-depleted mice differ from those of control mice (Figure 5, A and B). The number of $\mathrm{CD}^{+}$and $\mathrm{CD} 8^{+}$myocardial $\mathrm{T}$ cells was increased in the absence of $\mathrm{B}$ cells. This result is corroborated by previous literature suggesting that $\mathrm{B}$ cells regulate the trafficking of T cells in the context of tissue injury (24). B cell deficiency also altered myocardial monocytes, but the results observed were not consistent between antibody-mediated depletion and B cell deficiency due to a genetic mutation. This difference could be due to the fact that antibody-mediated B cell depletion causes an acute loss of B cells, whereas $\mu \mathrm{MT}$ mice are deprived of B cells throughout life. Another possible explanation is that $\mu \mathrm{MT}$ mice lack functional B1 cells, whereas antibody-mediated B cell depletion preserves B1 cells (2). It should also be noted that we did not measure the concentration of circulating leukocytes in B cell-deficient/depleted animals and controls. Although the organs studied were all carefully perfused, a difference in the circulating leukocyte numbers could have, to some extent, affected our findings.

We observed that B cell-deficient mice had smaller hearts, smaller myocardial fiber cross-sections, and higher left ventricular ejection fraction (Figure 5, C-E). These observations combined with our other results challenge the belief that leukocytes are not important for tissue homeostasis until they transition into the parenchyma (16). Myocardial B cells displayed upregulation of chemokine-chemokine signaling pathways and expressed several cytokines such as TGF- $\beta 1$ (Supplemental Figure 3), a known myocardial growth factor (25). Therefore, we propose that the effect of B cell ablation on myocardial mass might be due to an indirect paracrine communication between lymphocytes and cardiomyocytes. However, we studied myocardial mass in a genetic model of global B cell depletion ( $\mu \mathrm{MT}$ mice); therefore, our experiments do not allow us to differentiate between intracardiac versus extracardiac effects of B cells. Since $\mu$ MT mice have the same blood pressure as syngeneic controls (25), the observed difference in myocardial mass are unlikely to be due to variations in myocardial afterload, but other potential extracardiac effects of B cell depletion cannot be excluded.

Mice have been a powerful model to study immune systems. However, there are many discrepancies between murine and human immunity (26). Therefore, we also evaluated human heart samples and observed that the human heart harbors B cells that are primarily intravascular and in close contact with the endothelium (Figure 5, F and G). This result has the important limitation that it was collected from failing hearts and not naive hearts. However, it was consistent with our observations in the murine studies and suggests that our data might have relevance for human biology. Future studies will be needed to further evaluate these data using human myocardial B cells and heart tissue.

In summary, we demonstrated that the pool of myocardial B cells represents a subset of circulating B cells that adhere to the naive heart endothelium and arrest their transit as they pass through the heart. We found that the vast majority ( $>95 \%$ ) of myocardial B cells remain intravascular and few $(<5 \%)$ myocardial $\mathrm{B}$ cells cross the endothelium into myocardial tissue. We observed that myocardial B cells have distinct gene expression profiles compared with circulating B cells and are composed of B cells with different origins. By analyzing models of global B cell loss, we found that B cells play a significant role in modulating the myocardial leukocyte pool, as well as LV structure and function. These results unveil a previously unappreciated relationship between B lymphocytes and the heart. In addition, they show that subsets of naive B lymphocytes interact with the tissue vasculature and have an important physiological role even when they are not engaged in adaptive immune responses. We hypothesize that these observations highlight a previously unappreciated fundamental property of circulating B cells and that the current model of baseline B cell recirculation requires revision. Further research will be needed to verify the roles of myocardial B cells in antigen presentation and immune surveillance, to dissect the molecular basis of B cell effects on myocardial structure and function, and to establish whether our observations are relevant to B cell biology in other organs. 


\section{Methods}

Mice. We used 10- to 12-week-old female mice for all experiments unless otherwise specified. All mice were purchased from the Jackson Laboratory. The following strains were used: WT C57BL/B6J strain N. 000664, B6.129S2-Ighm ${ }^{\text {tm ICgn } / J ~(~} \mu \mathrm{MT}$ ) strain N.002288, B6.SJL-Ptprc ${ }^{a}$ Pepc ${ }^{b} /$ BoyJ (CD45.1) strain N. 002014, and B6.129S7-Rag1tm1Mom/J (Rag1) strain N. 002216. To generate CD19 reporter mice, B6.129P2(C)-Cd19tm1(cre)Cgn/J males (CD19-cre) were bred with B6.Cg-Gt(ROSA)26Sortm14(CAG-tdTomato)Hze/J females (tdTomato-lox), and F1 mice were studied. For antibody-mediated B cell depletion, 3-week-old WT mice were injected i.p. with $100 \mu \mathrm{g}$ of anti-CD20 antibody (BioLegend, clone SA271G2, catalog 152104) or isotype control. After 1 week, mice were reinjected retro-orbitally with $200 \mu \mathrm{g}$ of antiCD20 antibody or isotype control and thereafter reinjected with the same amount of antibody every 4 weeks for a total of 3 retro-orbital injections.

Parabiosis. Female donor and recipient mice were shaved, and matching skin incisions were made from behind the ear to the tail of each mouse. The s.c. fascia was dissected to create a free skin flap. The olecranon and knee joints were anastomosed using mono-nylon 5.0 suture (Ethicon), and skin flaps were anastomosed using a continuous suture. Postoperative anesthesia included $0.1 \mathrm{mg} / \mathrm{kg}$ s.c. injection of buprenorphine after surgery and 24 hours later. Mice received 3\% neomycin for 2 weeks and were analyzed at 6 weeks after surgery.

Intravital microscopy. Cardiac grafts were harvested from WT B6 mice and immediately transplanted into the right neck of B6 Cd19-tdTomato hosts as described previously (27). Cold ischemic time was $<30$ minutes. Intravital imaging of heart grafts was performed using a custom-built 2-photon microscope running ImageWarp version 2.1 software (A\&B Software) as described previously (27). Time-lapse imaging of lymphocyte trafficking in cardiac grafts was performed by averaging 15 video-rate frames $(0.5$ seconds per slice) captured during the acquisition to match the ventilator rate and minimize movement artifacts. Each plane represents an image measuring $220-240 \mu \mathrm{m}$ in the $x$ and $y$ dimensions. Twenty-one sequential planes were acquired in the $z$ dimension ( $2.5 \mu \mathrm{m}$ each) and compiled to generate a $Z$-stack. Coronary vessels were visualized by i.v. injection of $50 \mu \mathrm{L}$ of PBS containing $20 \mu \mathrm{L}$ of 655 -nm nontargeted Q-dots.

Heterotopic intraabdominal heart transplant. Heterotopic heart transplantation into the abdomen was performed as described previously (28). Briefly, animals were anesthetized with $100 \mathrm{mg} / \mathrm{kg}$ ketamine $\mathrm{HCl}$ and $10 \mathrm{mg} / \mathrm{kg}$ xylazine $\mathrm{HCl}$. Donor hearts were harvested en bloc, with a segment of the ascending aorta transected proximal to the innominate artery and a segment of the pulmonary artery transected close to its bifurcation. Then, donor hearts were implanted into the recipient abdominal cavity by end-to-side anastomoses of the donor's ascending aorta and pulmonary artery to the recipient's infrarenal aorta and inferior vena cava, respectively. Hearts were maintained on ice during the interval between harvest and implantation. The estimated time of cold ischemia was $\leq 1$ hour. The transplanted hearts were examined by daily check of pulsation until recipients were sacrificed.

Modified Langendorff perfusion. Hearts were isolated from heparinized $\mu \mathrm{MT}$ mice, immediately cannulated on ice, and then immediately transferred to a bath with modified Krebs-Henseleit buffer at $37^{\circ} \mathrm{C}$. Hearts were perfused at a constant pressure of $70 \mathrm{~mm} \mathrm{Hg}$ with modified Krebs-Henseleit buffer as described previously (29). After perfusion with $1 \mathrm{~mL}$ of buffer, hearts were perfused with $3 \mathrm{~mL}$ of a solution of heparinized blood freshly isolated from WT mice and Krebs-Henseleit buffer (1:1, v/v).

BM transplant and splenocyte adoptive transfer. BM was collected from the femur of donor mice. Recipient mice were lethally irradiated (10 Gy) and i.v. injected with 5 million BM cells collected on the same day as irradiation. Mice were analyzed for chimerism in the peripheral blood 3 weeks after irradiation and were consistently found to have $>95 \%$ chimerism. Hearts were collected for analysis 4 weeks after transplantation.

To perform BM transplant sparing the myocardium from irradiation, we used a small animal radiation research platform (SARRP, Xstrahl Inc.). To irradiate the femurs, mice were placed on the irradiation platform one at a time and fitted with a nose cone for isoflurane anesthesia. Cone beam computed tomography (CBCT) imaging was performed for each mouse to identify and pinpoint the femurs. The reconstructed CBCT images were imported into Muriplan (Xstrahl), which was used to select an isocenter on the femur. Then, the bilateral femurs were irradiated by administrating $10 \mathrm{~Gy}$ using anterior-posterior opposed beams. Mice were returned to the housing facility for postirradiation analyses.

For splenocyte adoptive transfer, the spleen of one 10-week-old CD45.1 WT mouse was minced and passed through a $40-\mu \mathrm{m}$ cell strainer. The cell solution was centrifuged, and RBCs were lysed in ACK lysis buffer (Invitrogen) for 15 minutes on ice. Splenocytes were resuspended in $200 \mu \mathrm{L}$ of sterile PBS and retro-orbitally injected in an age/sex-matched Rag1 mouse. 
Flow cytometry. For flow cytometry experiments, mice were euthanized in $\mathrm{CO}_{2}$ chamber. The hearts were perfused with cold PBS via high-pressure injection of $3 \mathrm{~mL}$ of PBS in the atria using a 3-mL syringe and an 18-gauge needle. Alternatively, hearts were retrogradely perfused with $2 \mathrm{~mL}$ of Krebs-Henseleit buffer after cannulation of the ascending aorta carefully dissected from extracardiac tissue under a stereo microscope, finely minced, suspended in $3 \mathrm{~mL}$ of DMEM, and digested with $120 \mathrm{U}$ of DNAse (MilliporeSigma), $180 \mathrm{U}$ of hyaluronidase (MilliporeSigma), and $1350 \mathrm{U}$ of collagenase (MilliporeSigma) for 60 minutes at $37^{\circ} \mathrm{C}$. The digested material was filtered through $40-\mu \mathrm{m}$ filters and pelleted by centrifugation $\left(250 \mathrm{~g}\right.$ for 3 minutes at $\left.4^{\circ} \mathrm{C}\right)$ in FACS buffer (PBS with 2\% FCS and 2 mM EDTA). RBCs were lysed in ACK lysis buffer (Invitrogen) for 5 minutes at room temperature, and the remaining cells were resuspended in $300 \mu \mathrm{L}$ of FACS buffer. Murine samples were labeled with the following fluorescently conjugated antibodies (from BioLegend): CD45 (clone 30-F11, PerCP/ Cy5.5 conjugated), CD19 (clone 1 D3/CD19, APC conjugated), CD11b (cone M1/70, BV510 conjugated or PE conjugated), IgM (clone RMM-1, Alexa Fluor 488 conjugated), CD5 (clone 53-7.3, PE/Cy7 conjugated), CD45.1 (clone A20, Pacific Blue conjugated), CD45.2 (clone 104, PerCP/Cy5.5 conjugated), Ly-6G (clone 1A8, FITC conjugated), CD64 (clone X54-5/7.1, PE conjugated), Ly-6C (clone HK1.4, APC/Cy7 conjugated), CD4 (clone GK1.5, PE conjugated), and CD8 (clone 53-6.7, Alexa Fluor 488 conjugated). Human samples were digested similarly as the murine samples and stained with the following antibodies: CD45 (clone H130, PE conjugated, Beckton Dickinson) and CD19 (clone HIB 19, APC conjugated, BioLegend). Cells were stained for 30 minutes on ice and washed in FACS buffer before analysis. All anti-murine antibodies from BioLegend were used at $0.2 \mu \mathrm{L}$ per $300 \mu \mathrm{L}$ sample, with the exception of the anti-CD45.2 antibody, which was used at $1 \mu \mathrm{L}$ per $300 \mu \mathrm{L}$. Anti-human antibodies were used at $1 \mu \mathrm{L} / 100 \mu \mathrm{L}$ dilution. FACS was performed using Becton Dickinson analyzers (LSRII, Canto, X20, or Fortessa). Compensation controls were generated using UltraComp ebeads (Invitrogen) and verified using single-color control samples obtained by staining primary splenocytes (only for murine samples). Gating strategies are summarized in Supplemental Figure 5. Cell sorting was performed using a FACSAria sorting instrument (Becton Dickinson) at the Washington University Department of Pathology Flow Cytometry and Sorting Core. In cell-sorting experiments, dead cells were excluded using the Live/Dead fixable aqua dye (BioLegend). Intravascular cells were labeled by diluting $10 \mu \mathrm{L}$ of antibody (20 $\mu \mathrm{g})$ in $150 \mu \mathrm{L}$ of sterile PBS, which was retro-orbitally injected approximately 3 minutes before sacrificing the mice.

Gravimetric and histological analyses. Mice were euthanized and hearts were removed and weighed to determine the heart weight/tibia length ratio. Then, hearts were processed, paraffin-embedded, and stained with WGA as described previously (30). For immunofluorescence analysis, $10 \mu \mathrm{m}$ of frozen heart section from CD19-tdTomato reporter hearts was stained with anti-CD31 primary antibody (polyclonal goat IgG, R\&D Systems, AF3628), followed by staining with a Northern Light 637 (R\&D Systems) conjugated secondary antibody. Sections were imaged using a Zeiss 880 Airyscan Fast 2-photon microscope or Olympus FV1000 2-photon microscope at the Washington University Center For Cellular Imaging (WUCCI).

For histological analysis of human heart, tissue collected and archived the time of implant of a left ventricular device was used. The H\&E-stained slides were reviewed to avoid area with extensive fibrosis. The CD20 stains were performed using Ventana Autostainer per manufacture's protocol (clone L26), with proper controls.

Echocardiographic studies. Ultrasound examination of the cardiovascular system was performed using a Vevo 2100 Ultrasound System (VisualSonics Inc.) equipped with a 30-MHz linear-array transducer as described previously (31).

Single cell transcriptional profiling. We used the Chromium Single Cell 3' v3 or 5' Library Kit and Chromium instrument (both from 10x Genomics) to perform single cell transcriptional profiling analyses. Approximately 17,500 CD19+ cells sorted from the heart or the blood of 3 mice were pooled together and partitioned into nanoliter droplets to achieve single cell resolution for a maximum of 10,000 individual cells per sample. The resulting cDNA was tagged with a common 16-nt cell barcode and 10- to 12-nt unique molecular identifier during the reverse transcription (RT) reaction. Fulllength cDNA from poly A-tailed mRNA transcripts was enzymatically fragmented and selected to optimize the cDNA amplicon size ( $400 \mathrm{bp}$ ) for library construction (10x Genomics). The single cell library concentration was quantified by quantitative PCR (qPCR) analysis (Kapa Biosystems) to produce appropriate cluster counts for the NovaSeq 6000 platform (Illumina). We generated 28-98 bp (3' v3 libraries) or 2-150 bp (5' libraries, V[D]J-enriched libraries, and TotalSeq libraries) sequence data targeting $\sim 50 \mathrm{~K}$ read pairs/cell for the gene expression library and $5 \mathrm{~K}$ read pairs/cell for the $\mathrm{V}(\mathrm{D})$ J-enriched or feature barcode library (TotalSeq), which provided digital gene expression profiles for each individual cell. 
Sample demultiplexing, barcode processing, and single cell 5' counting was performed using the Cell Ranger Single-Cell Software Suite (10x Genomics). Cell Ranger count was used to align samples to the reference genome ( $\mathrm{mm} 10)$, quantify reads, and filter reads with a quality score below 30 . The Seurat package in $\mathrm{R}$ was used for subsequent analysis (32). Cells with mitochondrial content greater than $0.05 \%$ were removed, and the cells that are duplets or mutliplets were filtered out. After removing the damaged cells, data were normalized using a scaling factor of 10,000 and were $\log$ transformed. The highly variable genes were selected using the FindVariableFeatures function with mean greater than 0.0125 or less than 3 and dispersion greater than 0.5 . These genes are used in performing the linear dimensionality reduction. Principal component analysis was performed before clustering, and the first 10 principal components (PCs) were used based on the ElbowPlot. Clustering was performed using the FindClusters function, which works on k-nearest neighbors (KNN) graph model with the granularity ranging from $0.1-0.9$ and selected 0.6 for the downstream clustering. For identifying the biomarkers for each cluster, we have performed differential expression between each cluster to all other clusters identifying negative and positive markers for that cluster. To generate DEGs, normalized cell-by-cell gene expression generated in previous steps was combined with sample data to separate gene expression data into samples. The linear normalized expression data for all cells in a given sample were averaged for each gene, resulting in a gene-by-sample matrix of "mean gene expression." To calculate fold change between circulating and myocardial B cells, the ratio of mean gene expressions for the respective sample was determined. To facilitate data interpretation, a pseudo-count of 0.0001 was added to both the numerator and denominator before determining the ratio to limit the effect of " 0 " values of mean gene expression on driving large fold changes. Thus, if a gene was not expressed in either genotype for a cluster, the fold change would be 1 . Using the MAST algorithm of the Seurat computation package in $\mathrm{R}$, the single cell expression distributions of 2 genotypes were compared for all cells, generating the $P$ value for differences between the 2 genotypes.

To address the possible confounding effect of tissue digestion on the comparison between myocardial and circulating B cells, the experiment was repeated after digesting the peripheral blood with the same enzymes used to digest the myocardium. This control experiment confirmed the differential gene expression observed between myocardial and circulating B cells. Pathway analysis of genes differentially expressed between myocardial and circulating B cells was performed using the GSEA software (17).

Statistics. Data are expressed as mean \pm SD. Two-tailed Student's $t$ test was used for pairwise comparisons between 2 groups, adjusting for multiple comparisons with the Benjamini-Hochberg procedure when appropriate. The specific statistical test used for each experiment is indicated in the figure legends. All analyses were performed using GraphPad Prism version 8. $P<0.05$ was used to determine statistical significance.

Study approval. All studies were performed with the approval of the IACUC at Washington University School of Medicine. These investigations conform to the Guide for the Care and Use of Laboratory Animals (National Academies Press, 2011).

\section{Author contributions}

LA designed the experiments, performed the experiments, and wrote the manuscript. DLM supervised the experiments and contributed to writing the manuscript. CRR performed experiments and assisted with data interpretation. SE performed the modified Langendorff perfusion. JW and GJR helped with BM transplant and data interpretation. $\mathrm{HD}$ and $\mathrm{KL}$ performed heterotopic heart transplants. CM and BER performed targeted animal irradiation. WL and DK performed intravital microscopy. CYL performed histological analysis of human myocardial tissue. MA and PSA performed single cell transcriptional profiling. All authors critically discussed the results, as well as edited and approved the final version of the manuscript.

\section{Acknowledgments}

The authors would like to acknowledge Marco Colonna and Ali Ellebedy for their thoughtful advice and their critical review of the manuscript and Deepta Bhattacharya for his thoughtful advice. This study was supported by research funds from the NIH (R01 HL-58081, HL-73017-0, HL-089543-01, HL 111094, R00 HL138163, T32 HL007081, S10OD020136, and 1K08HL145108-01A1), institutional funds from the Center for Cardiovascular Research at Washington University School of Medicine, and a pilot grant from the McDonnell Genome Institute at Washington University. The authors acknowledge the Washington University Digestive Disease Research Center Morphology Core (National Institute of Diabetes and Digestive and Kidney Diseases, P30 DK052574) for preparing the WGA histology specimens, and Erica Lantelme and the Washington 
University Flow Cytometry \& Fluorescence Activated Cell Sorting Core for assistance with FACS analysis and cell sorting. The manuscript was edited by the Scientific Editing Service supported by the Institute of Clinical and Translational Sciences at Washington University (NIH CTSA grant UL1 TR002345).

Address correspondence to: Douglas L. Mann or Luigi Adamo, Division of Cardiology, 660 S. Euclid Avenue, Campus Box 8086, St. Louis, Missouri 63110, USA. Phone: 314.362.8908; Email: dmann@wustl.edu (DLM); 1adamo@wustl.edu (LA).

1. Bönner F, Borg N, Burghoff S, Schrader J. Resident cardiac immune cells and expression of the ectonucleotidase enzymes CD39 and CD73 after ischemic injury. PLoS One. 2012;7(4):e34730.

2. Adamo L, et al. Modulation of subsets of cardiac B lymphocytes improves cardiac function after acute injury. JCI Insight. 2018;3(11):120137.

3. Yan X, et al. Temporal dynamics of cardiac immune cell accumulation following acute myocardial infarction. J Mol Cell Cardiol 2013;62:24-35.

4. Zouggari Y, et al. B lymphocytes trigger monocyte mobilization and impair heart function after acute myocardial infarction. Nat Med. 2013;19(10):1273-1280.

5. Cordero-Reyes AM, et al. Full Expression of Cardiomyopathy Is Partly Dependent on B-Cells: A Pathway That Involves Cytokine Activation, Immunoglobulin Deposition, and Activation of Apoptosis. J Am Heart Assoc. 2016;5(1):e002484.

6. Horckmans M, et al. Pericardial Adipose Tissue Regulates Granulopoiesis, Fibrosis, and Cardiac Function After Myocardial Infarction. Circulation. 2018;137(9):948-960.

7. Alberts B, et al. Lymphocytes and the Cellular Basis of Adaptive Immunity. New York, NY: Garland Science;2002.

8. Gowans JL, Knight EJ. The route of re-circulation of lymphocytes in the rat. Proc R Soc Lond, B, Biol Sci. 1964;159:257-282.

9. Cooper MD. The early history of B cells. Nat Rev Immunol. 2015;15(3):191-197.

10. Ramos GC, et al. Myocardial aging as a T-cell-mediated phenomenon. Proc Natl Acad Sci USA. 2017;114(12):E2420-E2429.

11. Pinto AR, et al. Revisiting Cardiac Cellular Composition. Circ Res. 2016;118(3):400-409.

12. Ray A, Dittel BN. Isolation of mouse peritoneal cavity cells. J Vis Exp. 2010;(35):1488.

13. Kitamura D, Roes J, Kühn R, Rajewsky K. A B cell-deficient mouse by targeted disruption of the membrane exon of the immunoglobulin mu chain gene. Nature. 1991;350(6317):423-426.

14. Sun H, Sun C, Xiao W, Sun R. Tissue-resident lymphocytes: from adaptive to innate immunity. Cell Mol Immunol. 2019;16(3):205-215.

15. Epelman S, et al. Embryonic and adult-derived resident cardiac macrophages are maintained through distinct mechanisms at steady state and during inflammation. Immunity. 2014;40(1):91-104.

16. Anderson KG, et al. Intravascular staining for discrimination of vascular and tissue leukocytes. Nat Protoc. 2014;9(1):209-222.

17. Subramanian A, et al. Gene set enrichment analysis: a knowledge-based approach for interpreting genome-wide expression profiles. Proc Natl Acad Sci USA. 2005;102(43):15545-15550.

18. Auffray C, et al. Monitoring of blood vessels and tissues by a population of monocytes with patrolling behavior. Science. 2007;317(5838):666-670.

19. Bhattacharya $\mathrm{D}$, et al. Transcriptional profiling of antigen-dependent murine B cell differentiation and memory formation. J Immunol. 2007;179(10):6808-6819.

20. Kin NW, Crawford DM, Liu J, Behrens TW, Kearney JF. DNA microarray gene expression profile of marginal zone versus follicular B cells and idiotype positive marginal zone B cells before and after immunization with Streptococcus pneumoniae. J Immunol. 2008;180(10):6663-6674.

21. Zikherman J, Parameswaran R, Weiss A. Endogenous antigen tunes the responsiveness of naive B cells but not T cells. Nature 2012;489(7414):160-164.

22. Kantor AB, Herzenberg LA. Origin of murine B cell lineages. Annu Rev Immunol. 1993;11:501-538.

23. Allman D, Lindsley RC, DeMuth W, Rudd K, Shinton SA, Hardy RR. Resolution of three nonproliferative immature splenic B cell subsets reveals multiple selection points during peripheral B cell maturation. J Immunol. 2001;167(12):6834-6840.

24. Chimen M, et al. Homeostatic regulation of T cell trafficking by a B cell-derived peptide is impaired in autoimmune and chronic inflammatory disease. Nat Med. 2015;21(5):467-475.

25. Dobaczewski M, Chen W, Frangogiannis NG. Transforming growth factor (TGF)- $\beta$ signaling in cardiac remodeling. $J$ Mol Cell Cardiol. 2011;51(4):600-606.

26. Mestas J, Hughes CC. Of mice and not men: differences between mouse and human immunology. J Immunol. 2004;172(5):2731-2738.

27. Li W, et al. Intravital 2-photon imaging of leukocyte trafficking in beating heart. J Clin Invest. 2012;122(7):2499-2508.

28. Zhang Z, Wang J-J, Wang X, Han J. Chapter 3. Kidney Transplantation in Mice. In, Chen H, Qian S, eds. Experimental Organ Trasnplantation. Waltham, Massachusetts, USA: Nova Medicine and Health; 2013.

29. Tzeng HP, et al. Dysferlin mediates the cytoprotective effects of TRAF2 following myocardial ischemia reperfusion injury. $J A m$ Heart Assoc. 2014;3(1):e000662.

30. Rocha-Resende C, et al. Immunomodulatory role of non-neuronal cholinergic signaling in myocardial injury. JCI Insight. 2019;5:128961.

31. Lavine KJ, Kovacs A, Weinheimer C, Mann DL. Repetitive myocardial ischemia promotes coronary growth in the adult mammalian heart. J Am Heart Assoc. 2013;2(5):e000343.

32. Butler A, Hoffman P, Smibert P, Papalexi E, Satija R. Integrating single-cell transcriptomic data across different conditions, technologies, and species. Nat Biotechnol. 2018;36(5):411-420. 